\title{
Clinical and histopathological features of a lingual mucosal horn: First time described clinical case series
}

\author{
Frédérick Gaultier ${ }^{1,3 *}$, Anne-Laure Ejeil ${ }^{2}$, Francois Lepelletier ${ }^{4}$, Bruno Gogly ${ }^{1,3}$, Hafida Cherifi ${ }^{1,3}$, Kinz Bayet ${ }^{1}$ and Sophie-Myriam Dridi $^{5}$ \\ ${ }^{1}$ Henri Mondor Hospital, Oral Medecine and Oral Surgery Department, Paris- Descartes University, Ile-de France, France \\ ${ }^{2}$ Bretonneau Hospital, Oral Medecine and Oral Surgery Department, Paris- Descartes University, Ile-de France, France \\ ${ }^{3}$ Laboratory of Molecular Oral Pathophysiology, INSERM 1138, Paris, France \\ ${ }^{4}$ Pitié Salpêtrière Hospital, Pathology Department, APHP and UPMC, Paris, France \\ ${ }^{5}$ Hopital Saint Roch Nice, Laboratoire Micoralis Microbiologie Orale, Immunotherapie et santé, EA 7354, UFR Odontologie Nice Cote d'azur 24 av des Diables \\ Bleus 06357 Nice Cedex 4, France
}

\begin{abstract}
Lingual mucosal horns are diagnostically challenging variants of a non-viral lingual papilloma that can clinically mimic the cutaneous horn. Improved characterization of these rare variants is needed, as improper diagnosis may lead to inappropriate or delayed treatment. A case series of patients with lingual horns diagnosed and treated at two academic hospitals from 2008 to 2019, was performed along with a literature review. We have diagnosed and inventoried seven lingual mucosal horns. The clinical description reveals a unique white overgrowth located on the dorsal surface of the tongue, delevoping at expense of the filiform papillae, without any preferential site but always located forward of the $\mathrm{V}$-shaped line. The lesions are firm but remain nevertheless flexible, movable, a few millimeters high, with a conical shape. The lingual mucosal horn is a conical overgrowth whose occurrence is extremely rare. The lingual mucosal horn is a non-viral papilloma, and differs from the cutaneous variant.
\end{abstract}

\section{Introduction}

The lingual horn is a conical overgrowth, located on the dorsal surface of the tongue, developing at the expense of a filiform papillae, whose occurrence is extremely rare. Laskaris describes this pathological entity for the first time in 1988 [1]. To our knowledge, this is the only author who has made a complete clinical description of the lingual horn. The systematic review carried out has indeed led to select only four articles. Some authors describe the only clinical features [1], others present the pathological analysis [2-4]. One of them was able to describe the clinical and a partial picture of the anatomopathological features [5]. Within the completeness of our systematic review, we are the first study to present a series of cases allowing us to clarify some of their clinical and histopathological characteristics of the lingual mucosal horn.

\section{Methods}

This study was conducted with patients from the oral mucosal pathologies' consultation of two separate hospital departments (Oral Pathology Department APHP, Henri Mondor Hospital - Créteil, Hôpital Bretonneau-Paris).

The cohort of patients is composed of six non-smoking women, aged 4 to 60 years, in good health except for a balanced type 2 diabetic female patient, and a healthy non-smoking male who is Caucasian. The five female patients are Caucasian, the other one is from Iran.

This systematic review was elaborated in accordance with the PRISMA guideline [6]. MEDLINE, Web of Science, Embase and Cochrane Database of Systematic Reviews search was conducted of all relevant literature until March 2019 using Mesh, and the following keywords: Lingual OR Tongue AND papilloma, Lingual OR Tongue AND Squamous papilloma, Lingual OR Tongue AND Horn Lingual OR Tongue AND Cornu. The electronic search was conducted independently by two authors (Frederick Gaultier, Sophie-Myriam Dridi).

Prior to 1935s, no publication was selected. Only full text articles published in English, French language have been considered.

\section{Inclusion criteria}

- Child and adult population

- Patients diagnosed with lingual papilloma, squamous papilloma, ligual horn.

\section{Exclusion criteria}

- No precise description of the clinical and or histopathological features.

Data were collected independently by two authors (Frederick Gaultier, Sophie-Myriam Dridi) and definitively included by these two authors (Frederick Gaultier, Sophie-Myriam Dridi), and all

${ }^{\star}$ Correspondence to: Frédérick Gaultier, DDS, PhD, Henri Mondor Hospital, Oral Medecine and Oral Surgery Department, Paris- Descartes University, Ilede France, France and Laboratory of Molecular Oral Pathophysiology, INSERM 1138, Paris, France, E-mail: frederick.gaultier@parisdescartes.fr

Key words: benign neoplasm, toungue, squamous cell papilloma

Received: April 17, 2019; Accepted: May 10, 2019; Published: May 15, 2019 
disagreements were resolved after discussion and/or with the intervention of a third author (Anne-Laure EJEIL).

All publications fulfilling the eligibility criteria were included, processed for data extraction and categorized according to the framework PICO:

Population: children and adult patients.

Intervention: horn, cornu, lingual, tongue.

Comparison: viral papilloma, squamous papilloma.

Outcome: first undescribed serial clinical cases with clinical and histopathological characteristics

The database search yielded 1585 references, including 954 from PubMed/MEDLINE, 491 from Web of Science, 140 from Embase, and 0 from Cochrane Database of Systematic Reviews. Following the selection of studies according to the inclusion and exclusion criteria and the removal of duplicate articles, full versions of 15 articles were selected for reading (Figure 1). After reading, 4 studies [2-5] met the inclusion criteria and were selected for the final analysis. Thus, 10 studies were excluded [7-15]; the reasons for exclusion are specified in Figure 1.

The conventional histology was completed by a dual immunhistochemistry staining Ki67 / p16 (Automate ventana benchmark ultra, closed system, compliance with manufacturer's standards, kit/ultraview, secondary antibody coupled to HRP multimer, primary antibodies Ki-67 pre-diluted 30-9 Roche (Ki67) and P16 INK4a pre-diluted E6H4 Roche (P16).

As only seven patients were treated, no statistical analyses comparing preoperative and postoperative values were conducted. As this is the first study, to our knowledge, to describe a clinical case series, the number of patients included is very small, thus we are not able to establish the prevalence of the lingual horns.

\section{Results}

From 2008 to 2019, we have diagnosed and inventoried seven lingual overgrowths compatible with the clinical criteria mentioned by Laskaris [1].

The clinical description reveals a unique white overgrowth located on the lingual dorsal surface, developing at expense of the filiform papillae, always located forward of the lingual V-shaped line. The lesions are firm but remain nevertheless flexible, movable, a few millimeters high, with a conical shape, well-defined and with a regular circular base, not indurated, resting on a normal lingual mucosa (Figures 2A and 2B).

The circumstances of appearance of the lingual horns could not have been determined. The lesions, all asymptomatic, are slowly growing which is compatible with a chronic process.
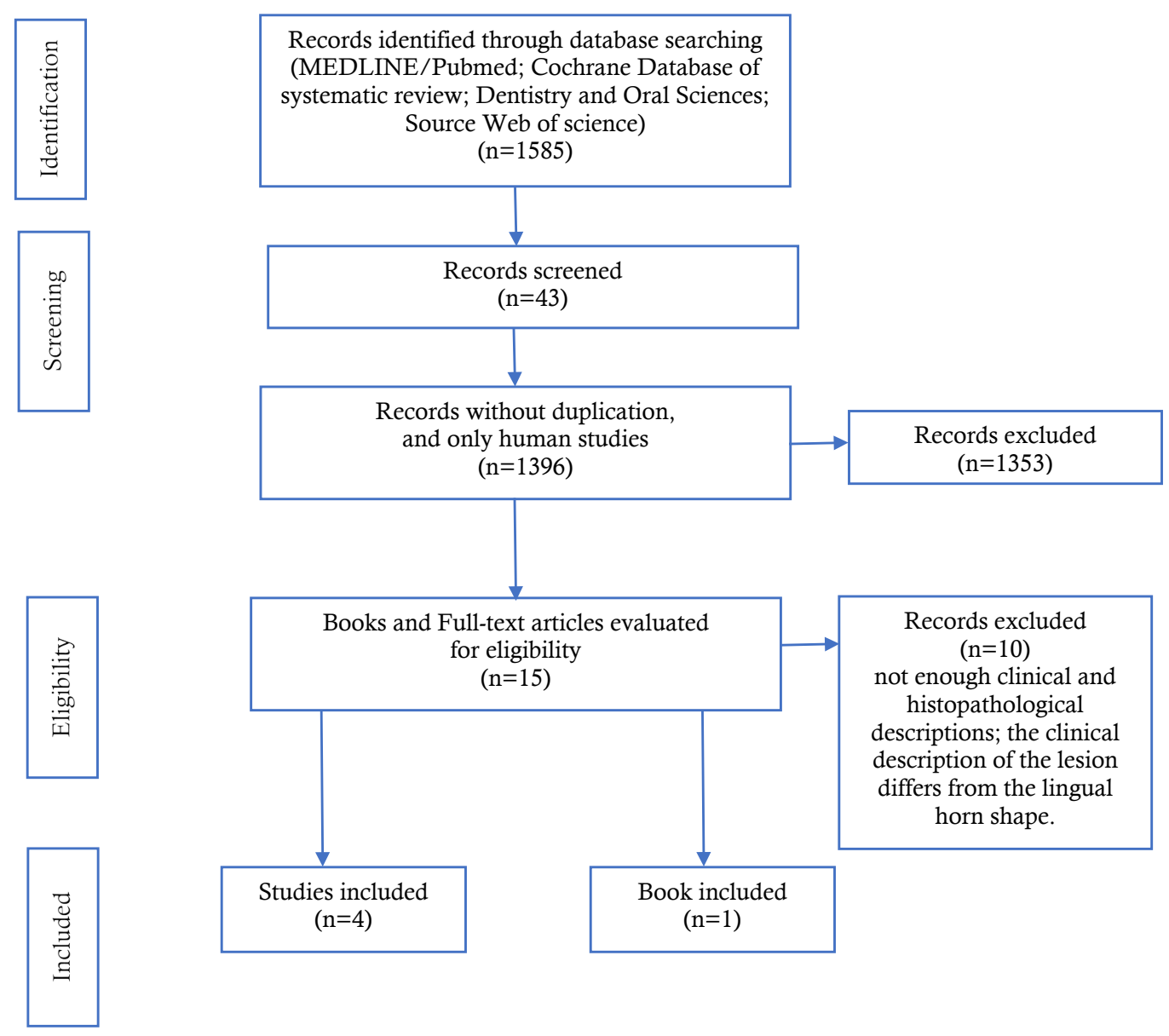

Figure 1. Flow diagram of the literature search and results 
The conventional histology (Figures $3 \mathrm{~A}$ and $3 \mathrm{~B}$ ) was completed by a dual immunhistochemistry staining (Ki67 / p16) because of the presence of some koilocyte-like cells within the epithelium (Figure 3C). Koilocytes are epithelial cells altered by a viral infection, they have a non-uniform perinuclear halo and a small and dense pycnotic nucleus. Although their presence is frequently associated with a viral infection, it does not represent a pathognomonic sign. Similarly, the absence of these cells does not formally rule out a viral etiology [16,17].

Exophytic papillomatosis is observed. No dysplasia was found (Figure 2B).

Surgical resection was performed without surgical margin under local anaesthesia: five were performed with cold blade and two with

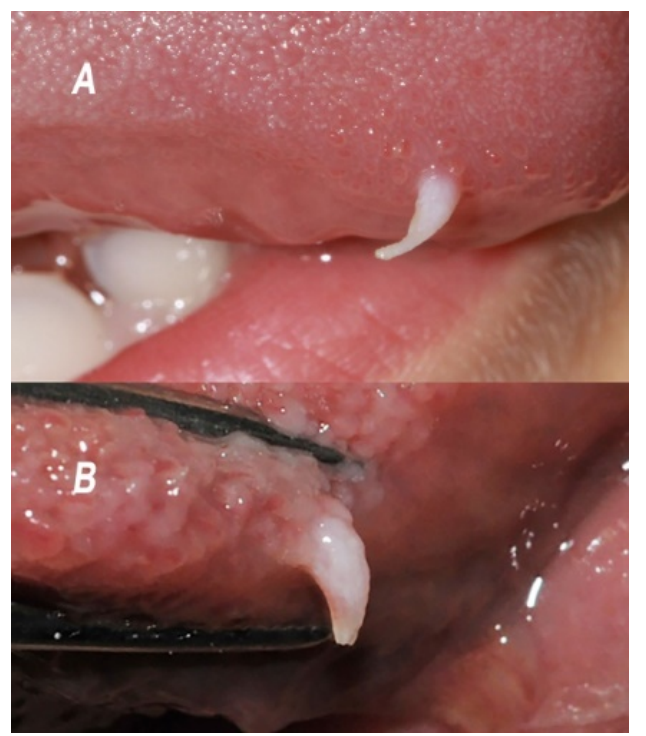

Figure 2. Lingual mucosal horn. Clinical aspect of two lingual horns, observed on the youngest (A) and the oldest (B) patients. These patients are in a general good health. The etiology is undetermined
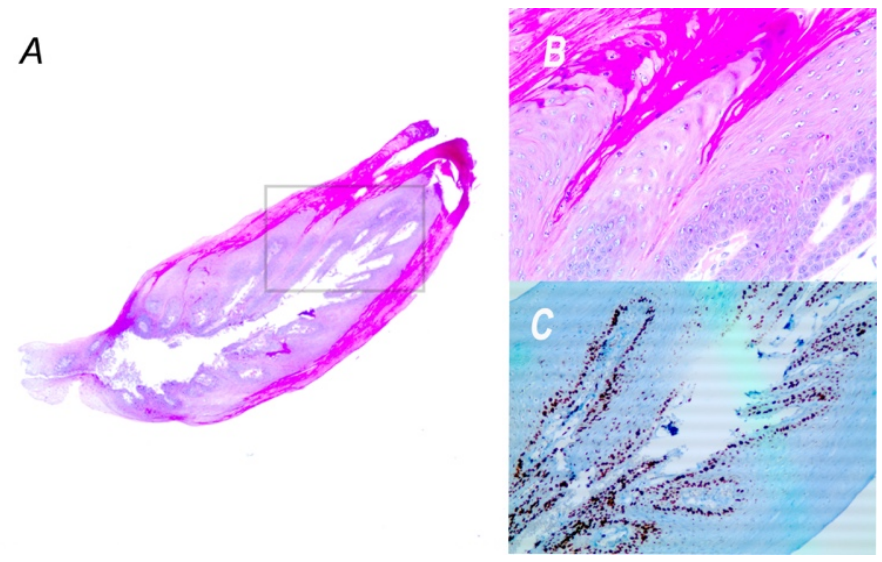

Figure 3. Histopathological section of the lingual mucosal horn. The haematoxylin and eosin staining shows a lesion that is higher than it is broad and a non-dysplastic hyperkeratotic squamous epithelium characterized by a centripetal exophytic hyperplasia of the epithelial ridges (A and B). The double immunohistochemistry (Ki67/p16) is negative. The Ki67 expression marks exclusively the basal cell (C). The overall results are consistent with a clinical diagnosis of non-viral papilloma.

A: HES Magnification x 25

B: HES Magnification x100 corresponding to the grey framed selection of the histological section.

C: Immunohistochemistry-Ki67 Magnification x200 corresponding to the grey framed selection of the histological section
$\mathrm{CO}_{2}$ laser [18] (4W, CW), without any surgical outcomes. An antiseptic mouthwash was prescribed for three days (Chlorexhidine $0.12 \%$ ).

\section{Discussion}

In the small number of follow-up patients of this cohort, it seems that there are more women with lingual horns than men, without it being possible to assert that there is a genetic predisposition.

This type of injury can occur at any age, with non-smoking patients and in a good general health. The clinical form is similar to one observed for cutaneous horns [19-21] (Figure 4), especially those observed on the lips [20], presenting a firm conical growth but whose keratosis actinic growth can evolve towards a malignant transformation [21]. To our clinical experience, lingual mucosal horn has no potential malignity, nevertheless the histopathological analysis has to be performed to establish the diagnostic.

The typical appearance is a non-viral papilloma which is developing at the expense of filiform papillae. It is likely a traumatic origin [2].

The differential diagnosis must be done: a circumscribed and exophytic sessile elevations that characterize viral papillomas $[2,3,8]$ and condylomas [22,23] caused by HPV (Human Papilloma Virus). The viral papillomas and condylomas have thus a stocky and massive appearance characterized by a typically papillomatous surface (Figure $5 \mathrm{~A}$ ), far different from the conical shape described below. As child's condyloma impose to determine if the child was sexually abused, it's underlined the importance to distinguish the non-viral lingual mucosal horn from the viral papillomas $[2,3,8]$ and condylomas [23]. One authors describe the lingual horns'clinical features in a pediatric descriptive article [7] which is not described in other books [24] and clinical reviews [14,25]. The clinical diagnosis is easy if the lesions are multiple; nevertheless, this requires the result of the pathological examination: vacuolated cytoplasm of balloon cells with pyknotic nucleus with a non-uniform perinuclear halo whose anatomopathological appearance of koilocytes may indicate the intracellular presence of virions.

Despite the presence of koilocytes and if there is any doubt the viral etiology, Ki67/p16 immunohistochemistry double staining can be routinely performed to rule out HPV infection. Initially, this double staining was developed to detect HPV infections, associated with cellular dysplasia, in cervical squamous epithelia; its sensitivity is comparable to the HPV test, with a better specificity [26]. Immunohistochemistry Ki67 (IHC Ki67) marks the cells during the cycle, not only those in mitosis [27]. It is a marker of proliferation, sensitive and specific to a dysplasia within a squamous epithelium. On one hand, a positive Ki67 (Ki67+) is defined by the presence of at least two strongly marked nuclei in the $2 / 3$ upper part of the epithelial layer which is generally associated with the presence of HPV DNA [17]; on the other hand, a negative Ki67 (KI67-) represents a typically basal staining which has been observed for the lingual horn of the young 4 years' old patient (Figure 3C).

The viral protein p16, produced by HPV type 16 viruses, is a molecular biomarker whose overexpression indirectly reflects the overexpression of the viral E7 oncogene. This oncogene targets the protein expressed by the $\mathrm{pRb}$ gene leading to a negative feedback on the repression of the transcription of the p16 gene, which results in an increasing translation of this protein within the infected cells. IHC P16 is positive when diffuse staining is intense at least on the $2 / 3$ lower part of the squamous epithelium [17,28]. For the 4-year-old child lingual horn, IHC P16 is negative. 


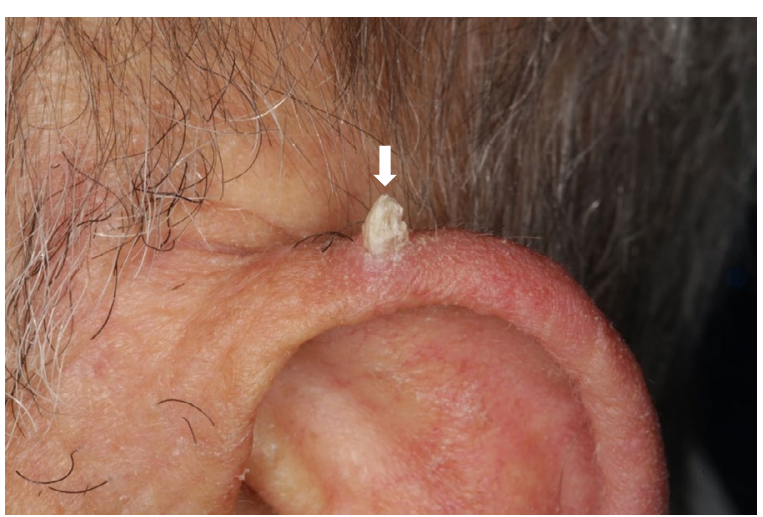

Figure 4. Cutaneous horn. The cutaneous horn is located on a on 70-year-old man's pinna, whose keratosis actinic growth can evolve towards a malignant transformation

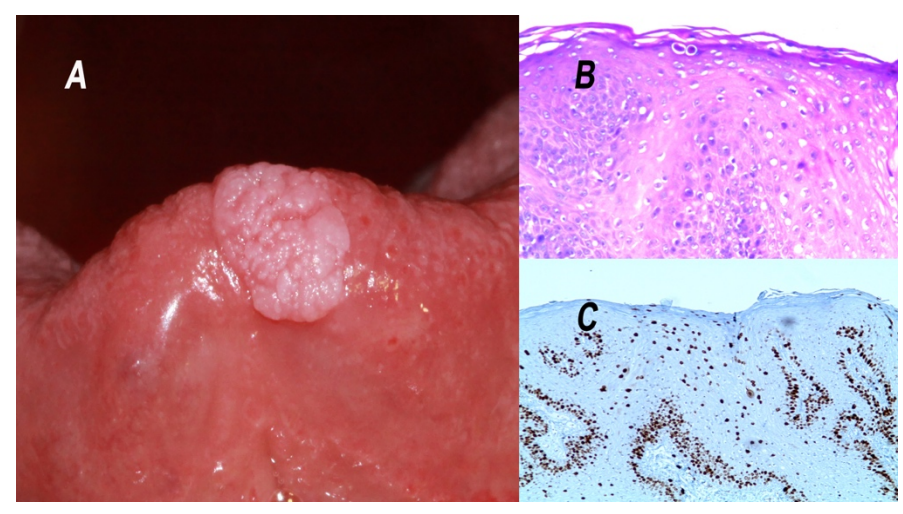

Figure 5. Lingual condyloma - Histopathological sections.

A: Lingual condyloma.

B: Histopathological section of the lingual condyloma. Magnification x200

C: Immunohistochemistry-Ki67 Magnification x200

The condyloma represents the main differential diagnosis of the lingual horn. However, its appearance is different: exophytic, whitish, with a papillomatous cauliflower surface or a cockscombs aspect (A). Its histological characteristics are also distinct: short epithelial ridges with many koilocytes located in the spinous layer (B) which is confirmed by a layered positive Ki67 staining $(C)$. In this case, the absence of cellular atypia and mitoses at a distance from the basal layer, argues against cellular dysplasia. The p16 expression is negative (data not shown). These results are consistent with the diagnosis of condyloma

Therefore, conventional histological analysis coupled with p16 / Ki67 double immunohistochemistry staining is compatible with the diagnosis of non-viral papilloma $[2,3,8]$.

Given the morphology of the lingual horn, the differential diagnosis is usually easy. Oral papules that appear during Heck's disease can be ruled out immediately; these papules are always multiple, located on the labial and jugal mucosa, these are soft and depressible lesions, rather pink than white. This differential diagnosis can however be considered with the vegetations induced by the human papilloma virus which can also lookalike asymptomatic unique, sessile, firm and supple papules, more or less white which depends on the amount of keratinization.

Common wart, induced by HPV 2-4-6, are readily observed on the dry vermillon of the lips and the dorsal anterior part of the tongue. Warts are round or oval papules with a finely roughened surface and smaller than lingual horns. Their presence coincides usually with palmar warts. This phenomenon of self-inoculation facilitates the establishment of the diagnosis of certainty [29]. These warts are characterized by elongated rete ridges which tend to converge toward the center of lesion (cupping effect), a thickened granular layer (hypergranulosis) with aggregated keratohyaline granules, and abundant koilocytes in a IHC Ki67 positive superficial epithelial spinous layer.

Viral papillomas and condylomas of comparable clinical appearance can be isolated in immunocompetent patients without other concomitant mucocutaneous lesions. Oral viral papillomas are generated by HPV 6 and 11 and condylomas mainly by HPV 16, but also by HPV 18 and 31 . However, their particularities can differentiate them from lingual horns. These lesions are generally expressed by exophytic fleshy masses, pink to whitish, with a papillomatous cauliflower surface or a cockscombs aspect (Figure 5A). The epithelial ridges are short, more or less sharps with many koilocytes in the spinus layer (Ki67+) (Figures 5B and 5C).

In conclusion, the histological analysis of all lesions of the clinical serie is compatible with the non-viral papilloma characteristics as described by Fronie et al. and Carneiro et al. [2,3].

The diagnosis of benign lingual tumors is easily done. The lingual horn is not a pathological entity but represents a morphological variant of a non-viral papilloma specific to the tongue. Therefore, patients should be reassured that not to be anxious. These are benign, noncontagious lesions that can be easily removed with a cold blade or the $\mathrm{CO}_{2}$ laser [18] whose haemostatic action requires no suture. As the aetiology is non-viral, therefore the carbonization vapours contain no virions. No suture removal is needed. Ad integrum healing is obtained from the first week. The absence of sign correlated to the gravity does not impose any perilesional surgical margin. The complete surgical resection is not at risk of recurrence.

We highlight key features of lingual horns proposed by the scientific literature as diagnostic criteria; however, case reviews are limited by publication biais of poor outcomes, near misses, or missing information that affects results. International multicentric studies are needed to validate the proposed features as diagnostic criteria for lingual mucosal horns. Future research is especially needed to to confirm their non capacity to a malignant transformation. In the limit of the small number of cases described, the results have to be confirmed by carrying out additional studies.

\section{Conclusions}

This cases series is, to our knowledge, the first one describing the lingual mucosal horns which have not been published in the scientific literature. The lingual horn is not a pathological entity but represents a morphological variant of a non-viral papilloma specific to the tongue. This work thus provides an iconographic support to accompany the diagnostic procedure from the clinical examination to the pathological examination. The simple care for patients which is accessible to all practitioners is minimally invasive and as safe as possible.

\section{Declarations}

Author contributions: Drs Dridi, Ejeil and Gaultier had full access to all of the data in the study and take responsibility for the integrity of the data and the accuracy of the data analysis.

\section{Concept and design: Dridi, Gaultier.}

Acquisition, analysis, or interpretation of data: Dridi, Ejeil, Gaultier, Le Pelletier.

\section{Drafting of the manuscript: Gaultier, Dridi.}

Critical revision of the manuscript for important intellectual content: All authors. 
Administrative, technical, or material support: Bayet, Cherifi, Dridi, Ejeil, Gaultier, Le Pelletier.

\section{Supervision: Dridi, Ejeil, Gaultier, Gogly.}

conflict of interests: None reported.

\section{References}

1. Laskaris G (1988) Color atlas of oral diseases. Stuttgart: Thieme, pp : 321.

2. Fronie A, Dumitrescu D, Vască V, Fronie A (2011) Histopathological aspects of benign epithelial tumors located in areas of friction or chronic irritation of the tongue. Romanian J Morphol Embryol Rev Roum Morphol Embryol 52: 831-835.

3. Carneiro TE, Marinho SA, Verli FD, Mesquita ATM, Lima NL, et al. (2009) Oral squamous papilloma: clinical, histologic and immunohistochemical analyses. $J$ Oral Sci 51: 367-372.

4. Henley JD, Summerlin DJ, Tomich CE (2004) Condyloma acuminatum and condylomalike lesions of the oral cavity: a study of 11 cases with an intraductal component. Histopathology 44: 216-221.

5. Ottoman BAE (2015) Squamous papilloma of the tongue: A case report. Int J Sci Rep 1: 163 .

6. Moher D, Liberati A, Tetzlaff J, Altman DG, The PRISMA Group (2009) Preferred reporting items for systematic reviews and meta-analyses: The PRISMA statement. PLoS Med 6: e1000097.

7. Michel B, Couly G (2004) Tumeurs et dysplasies tumorales de la cavité buccale du nouveau-né et du nourrisson. EMC-Dent. Août 1: 214-227.

8. Frigerio M, Martinelli-Kläy CP, Lombardi T (2015) Clinical, histopathological and immunohistochemical study of oral squamous papillomas. Acta Odontol Scand 73: 508-515.

9. Jaju PP, Suvarna PV, Desai RS (2010) Squamous papilloma: case report and review of literature. Int J Oral Sci 2: 222-225. [Crossref]

10. Rose HP (1965) Papillomas of the oral cavity. Oral Surg Oral Med Oral Pathol 20: 542-549. [Crossref]

11. Gullifer WH (1938) Papilloma. Am J Orthod Oral Surg 24: 884-886.

12. Colon PG Jr (1980) Oral papilloma in marijuana users. Quintessence Int Dent Dig 11: 75-80. [Crossref]

13. Thomas GJ, Barrett AW (2009) Papillary and verrucous lesions of the oral mucosa. Diagn Histopathol 15: 279-285.

14. Horn C, Thaker HM, Tampakopoulou DA, Serres LMD, Keller JL, et al. (2001) Tongue lesions in the pediatric population. Otolaryngol-Head Neck Surg 124: 164-169.
15. Orlean SL (1956) Papilloma of tongue; report of a case. Oral Surg Oral Med Oral Pathol 9: 937-938. [Crossref]

16. Miyahara GI, Simonato LE, Mattar NJ, Camilo DJ, Biasoli ER (2011) Correlation between koilocytes and human papillomavirus detection by PCR in oral and oropharynx squamous cell carcinoma biopsies. Mem Inst Oswaldo Cruz 106: 166-169.

17. Pirog EC (2015) Immunohistochemistry and in situ hybridization for the diagnosis and classification of squamous lesions of the anogenital region. Semin Diagn Pathol 32: 409-418.

18. White JM, Chaudhry SI, Kudler JJ, Sekandari N, Schoelch ML, et al. (1998) Nd:YAG and CO2 laser therapy of oral mucosal lesions. J Clin Laser Med Surg 16: 299-304. [Crossref]

19. Yu RC, Pryce DW, Macfarlane AW, Stewart TW (1991) A histopathological study of 643 cutaneous horns. Br J Dermatol 124: 449-452. [Crossref]

20. Monaghan AM (1993) Cutaneous horn occurring on the vermilion border of the lower lip. Br Dent $J$ 175: 419-420. [Crossref]

21. Phulari RG, Rathore R, Talegaon TP, Shah A (2018) Cutaneous horn: A mask to underlying malignancy. J Oral Maxillofac Pathol 22: S87-87S90. [Crossref]

22. Zunt SL, Tomich CE (1989) Oral condyloma acuminatum. J Dermatol Surg Oncol 15 591-594. [Crossref]

23. Percinoto ACC, Danelon M, Crivelini MM, Cunha RF, Percinoto C (2014) Condyloma acuminata in the tongue and palate of a sexually abused child: a case report. BMC Res Notes 7.

24. Philipone E, Yoon AJ (2017) Oral pathology in the pediatric patient: A clinical guide to the diagnosis and treatment of mucosal lesions. Springer, pp: 148

25. Bouquot JE, Adibi SS, Sanchez M (2012) Chronic lingual papulosis: new, independent entity or "mature" form of transient lingual papillitis? Oral Surg Oral Med Oral Pathol Oral Radiol 113: 111-117.

26. Schmidt D, Bergeron C, Denton KJ, Ridder R (2011) For the European CINtec Cytology Study Group (2011) p16/ki-67 dual-stain cytology in the triage of ASCUS and LSIL Papanicolaou cytology: Results from the European Equivocal or Mildly Abnormal Papanicolaou cytology study. Cancer Cytopathol 119: 158-166.

27. Gerdes J, Lemke H, Baisch H, Wacker HH, Schwab U, et al. (1950) Cell cycle analysis of a cell proliferation-associated human nuclear antigen defined by the monoclonal antibody Ki-67. J Immunol Baltim Md 133: 1710-1715.

28. Lim S, Lee MJ, Cho I, Hong R, Lim SC (2016) Efficacy of p16 and Ki-67 immunostaining in the detection of squamous intraepithelial lesions in a high-risk HPV group. Oncol Lett 11: 1447-52.

29. Betz SJ (2019) HPV-Related Papillary Lesions of the Oral Mucosa: A Review. Head Neck Pathol 13: 80-90. [Crossref]

Copyright: (C2019 Gaultier F. This is an open-access article distributed under the terms of the Creative Commons Attribution License, which permits unrestricted use, distribution, and reproduction in any medium, provided the original author and source are credited. 